
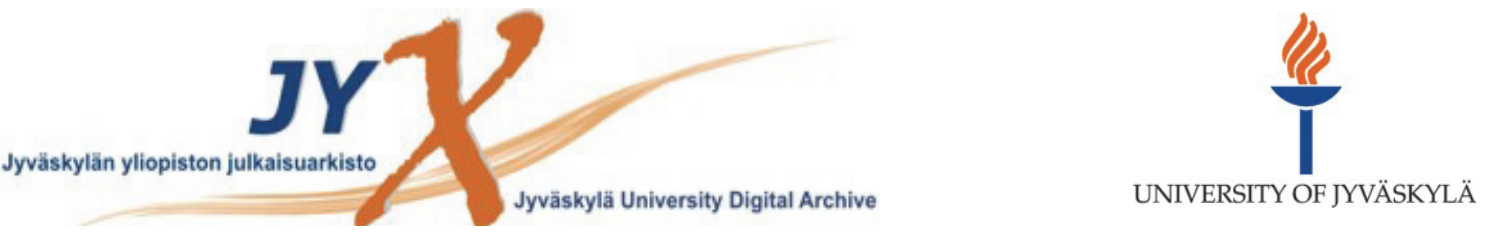

This is an electronic reprint of the original article. This reprint may differ from the original in pagination and typographic detail.

\author{
Author(s): Agramunt, J.; Tain, J.L.; Gómez-Hornillos, M.B.; Garcia, A.R.; Albiol, F.; Algora, A.; \\ Caballero-Folch, R.; Calviño, F.; Cano-Ott, D.; Cortés, G.; Domingo-Pardo, C.; Eronen, \\ Tommi; Gelletly, W.; Gorelov, Dmitry; Gorlychev, V.; Hakala, Jani; Jokinen, Ari; Jordan, \\ M.D.; Kankainen, Anu; Kolhinen, Veli; Kucuk, L.; Martinez, T.; Mason, P.J.R.; Moore, \\ lain; Penttilä, Heikki; Podolyák, Zs.; Pretel, C.; Reponen, Mikael; Riego, A.; Rissanen, \\ Title: Characterization of a neutron-beta counting system with beta-delayed neutron \\ emitters
}

Year: $\quad 2016$

Version:

Please cite the original version:

Agramunt, J., Tain, J.L., Gómez-Hornillos, M.B., Garcia, A.R., Albiol, F., Algora, A., Caballero-Folch, R., Calviño, F., Cano-Ott, D., Cortés, G., Domingo-Pardo, C., Eronen, T., Gelletly, W., Gorelov, D., Gorlychev, V., Hakala, J., Jokinen, A., Jordan, M.D., Kankainen, A., ... Valencia, E. (2016). Characterization of a neutron-beta counting system with beta-delayed neutron emitters. Nuclear Instruments and Methods in Physics Research Section A : Accelerators, Spectrometers, Detectors and Associated Equipment, 807, 69-78. https://doi.org/10.1016/j.nima.2015.10.082

All material supplied via JYX is protected by copyright and other intellectual property rights, and duplication or sale of all or part of any of the repository collections is not permitted, except that material may be duplicated by you for your research use or educational purposes in electronic or print form. You must obtain permission for any other use. Electronic or print copies may not be offered, whether for sale or otherwise to anyone who is not an authorised user. 


\title{
Characterization of a Neutron-Beta Counting System With Beta-Delayed Neutron Emitters
}

J. Agramunt ${ }^{\mathrm{a}}$, J.L. Tainn ${ }^{\mathrm{a}, *}$, M.B. Gómez-Hornillos ${ }^{\mathrm{b}}$, A.R. Garcia ${ }^{\mathrm{c}}$, F. Albiol ${ }^{\mathrm{a}}$, A. Algora ${ }^{\mathrm{a}, \mathrm{g}}$, R. Caballero-Folch ${ }^{\mathrm{b}}$, F. Calviño ${ }^{\mathrm{b}}$, D. Cano-Ott $^{\mathrm{c}}$, G. Cortés ${ }^{\mathrm{b}}$, C. Domingo-Pardo ${ }^{\mathrm{a}}$, T. Eronen ${ }^{\mathrm{d}}$, W. Gelletly ${ }^{\mathrm{e}}$, D. Gorelov ${ }^{\mathrm{d}}$, V. Gorlychev ${ }^{\mathrm{b}}$, H. Hakala ${ }^{\mathrm{d}}$, A. Jokinen ${ }^{\mathrm{d}}$, M.D. Jordan $^{\mathrm{a}}$, A. Kankainen ${ }^{\mathrm{d}}$, V. Kolhinen ${ }^{\mathrm{d}}$, L. Kucuk ${ }^{\mathrm{f}}$, T. Martinez ${ }^{\mathrm{c}}$, P.J.R. Mason ${ }^{\mathrm{e}}$, I. Moore ${ }^{\mathrm{d}}$, H. Penttilä ${ }^{\mathrm{d}}$, Zs. Podolyák $^{\mathrm{e}}$, C. Pretel $^{\mathrm{b}}$, M. Reponen ${ }^{\mathrm{d}}$, A. Riego ${ }^{\mathrm{b}}$, J. Rissanen $^{\mathrm{d}}$, B. Rubio ${ }^{\mathrm{a}}$, A. Saastamoinen ${ }^{\mathrm{d}}$, A. Tarifeño-Saldivia ${ }^{\mathrm{a}, \mathrm{b}}$, E. Valencia $^{\mathrm{a}}$

\author{
${ }^{a}$ Instituto de Física Corpuscular, CSIC - Universidad de Valencia, E-46071 Valencia, Spain \\ ${ }^{b}$ Secció d'Enginyeria Nuclear, Universitat Politecnica de Catalunya, E-08028 Barcelona, Spain \\ ${ }^{c}$ Centro de Investigaciones Energéticas Medioambientales y Tecnológicas, E-28040 Madrid, Spain \\ ${ }^{d}$ Department of Physics, University of Jyväskylä, FI-40014 Jyväskylä, Finland \\ ${ }^{e}$ Department of Physics, University of Surrey, Guildford GU2 7XH, UK \\ ${ }^{f}$ Department of Physics, University of Istanbul, 34134 Vezneciler, Turkey \\ ${ }^{g}$ Institute of Nuclear Research of the Hungarian Academy of Sciences, 4001 Debrecen, Hungary
}

\begin{abstract}
A new detection system for the measurement of beta-delayed neutron emission probabilities has been characterized using fission products with well known $\beta$-delayed neutron emission properties. The setup consists of BELEN-20, a $4 \pi$ neutron counter with twenty ${ }^{3} \mathrm{He}$ proportional tubes arranged inside a large polyethylene neutron moderator, a thin Si detector for $\beta$ counting and a selftriggering digital data acquisition system. The use of delayed-neutron precursors with different neutron emission windows allowed the study of the effect of energy dependency on neutron, $\beta$ and $\beta$-neutron rates. The observed effect is well reproduced by Monte Carlo simulations. The impact of this dependency on the accuracy of neutron emission probabilities is discussed. A new accurate value of the neutron emission probability for the important delayed-neutron precursor ${ }^{137} \mathrm{I}$ was obtained, $P_{n}=7.76(14) \%$.
\end{abstract}

Keywords:

Beta-delayed neutron emission probability, Neutron and beta counters, Trigger-less digital data acquisition system, Geant4 simulations

\section{Introduction}

Beta-delayed neutron emission is a form of decay that occurs for nuclei with a large enough neutron excess. For delayedneutron precursors the neutron separation energy in the daugh5 ter nucleus $S_{n}$ is smaller than the decay energy window $Q_{\beta}$. As a result neutron unbound states can be populated in the decay. The process becomes dominant far enough from stability. The $\beta$-delayed neutron emission probability $P_{n}$ conveys information about the distribution and nature of nuclear levels populated in the decay, which is dictated by nuclear shell structure and residual interactions [1]. $P_{n}$ values are also important inputs for the description of the astrophysical $r$ process responsible for the synthesis of about half of the observed abundance of elements heavier than iron [2]. These two fields of interest, nuclear structure and nuclear astrophysics, explain why the measurement of $P_{n}$ values for exotic nuclei is one of the goals of the DEcay SPECtroscopy (DESPEC) experiment [3] within the NUclear STructure and Astrophysics Research (NUSTAR) collaboration [4] at the Facility for Antiproton and Ion Research (FAIR) [5]. The BEta deLayEd Neutron (BELEN) counter has been developed [6] for this purpose. It is based on the well

*Tel.: +34 963543497, Fax: +34 963543488. Instituto de Física Corpuscular, Apdo. Correos 22085, E-46071 Valencia, Spain

Email address: tain@ific.uv.es (J.L. Tain) proven technology $[7,8,9,10,11,12]$ of combining an array of ${ }^{3} \mathrm{He}$ proportional tubes, selectively sensitive to low energy neutrons, with a hydrogenous neutron energy moderator. Detectors with large solid angle and detection efficiency can be built in this way. The BELEN detector was conceived as a flexible and easily reconfigurable system. The current version has fortyeight ${ }^{3} \mathrm{He}$ tubes, but previous versions with twenty [13, 14] and thirty [15] tubes have been used in different measurements. Some of these measurements were aimed at the accurate determination of $P_{n}$ values of fission products relevant in reactor technology. The fraction of neutrons in the reactor core coming from $\beta$ decay is an important parameter for the safe control of reactor power [16]. This constitutes the third field of application of this detector. The BELEN detector is part also of the largest neutron counter of this kind (more than $160{ }^{3} \mathrm{He}$ tubes) that is being assembled by the BRIKEN collaboration [17] for the measurement of exotic nuclei at RIKEN.

In this work we describe the characterization of the detector setup installed at the Cyclotron Laboratory of the University of Jyväskylä during the 2010 measuring campaign [14]. For the characterization of the setup we used fission products which are delayed-neutron precursors with well known properties. The instrumentation includes BELEN-20 and a Si $\beta$ detec4 tor, a fairly common arrangement. A novelty in our setup is the introduction of a trigger-less data acquisition system. Its use al- 
lows continuous control of data quality, which leads to greater accuracy, with a minimum acquisition dead time. In this work we also discuss some of the systematic effects which appear in the use of $\beta$-neutron counting systems applied to the determination of $P_{n}$ values.

\section{Determination of $\boldsymbol{P}_{\boldsymbol{n}}$ values}

The $P_{n}$ value is the fraction of all decays which undergo ${ }^{95}$ delayed-neutron emission. It is a common experimental approach, and the one we follow here, to obtain the number of decays from the number of $\beta$ particles registered in a $\beta$ detector and the number of $\beta$-delayed neutron decays from the number of neutrons observed in a neutron detector. Taking into account ${ }^{100}$ the fact that detection efficiencies for both $\beta$ particles and neutrons are energy dependent we can write

$$
P_{n}=\frac{\sum_{i, E_{i}>S_{n}} \sum_{f} \frac{N_{n}^{i f}}{I_{\beta}^{i} I_{n}^{i f} \varepsilon_{n}^{i f}}}{\sum_{i} \frac{N_{\beta}^{i}}{I_{\beta}^{i} \varepsilon_{\beta}^{i}}}=\frac{\bar{\varepsilon}_{\beta}}{\bar{\varepsilon}_{n}} \frac{N_{n}}{N_{\beta}}
$$

In this equation $i$ designates a level in the daughter nucleus 110 ${ }^{Z+1} A$ at excitation energy $E_{i}$ populated with probability $I_{\beta}^{i}$, and $f$ a level in the final nucleus ${ }^{Z+1} A-1$ at excitation energy $E_{f}$, which is populated from level $i$ with probability $I_{n}^{i f} . N_{n}^{i f}$ is the 65 number of detected neutrons with an efficiency $\varepsilon_{n}^{i f}$, which are emitted in the transition $i \rightarrow f$ with energy $E_{n}^{i f}=E_{i}-E_{f}-S_{n} \cdot{ }^{115}$ $N_{\beta}^{i}$ is the number of detected $\beta$ particles with an efficiency $\varepsilon_{\beta}^{i}$, which are emitted in the decay of the parent nucleus ${ }^{Z} A$ to level $i$. The right-hand side of Eq. 1 is the expression commonly 70 employed to calculate the $P_{n}$ value but it emphasizes that $\bar{\varepsilon}_{\beta}$ and $\bar{\varepsilon}_{n}$ are average $\beta$ and neutron efficiencies for all $\beta$ particles and all neutrons. Note that the summation over levels in the daughter nucleus is restricted to neutron unbound states $\left(E_{i}>_{120}\right.$ $S_{n}$ ) in the numerator of Eq. 1, but runs over all levels (including

75 the ground state) in the denominator. From the form of Eq. 1 it is clear that the average efficiencies are nuclide dependent. Equation 1 assumes that only one neutron is emitted per decay. For multiple neutron emission appropriate formulae for $P_{x n} \mathrm{can}_{125}$ be written.

80 Sometimes the quantity measured is the number of neutrons in coincidence with the $\beta$ particle. This is necessary to enhance the neutron detection sensitivity whenever the rate of $\beta$ delayed neutrons is comparable to or smaller than the rate of ${ }_{130}$ background neutrons. In this case the expression reads

$$
P_{n}=\frac{\sum_{i, E_{i}>S_{n}} \sum_{f} \frac{N_{\beta n}^{i f}}{I_{\beta}^{i} I_{n}^{i f} \varepsilon_{\beta}^{i} \varepsilon_{n}^{i f}}}{\sum_{i} \frac{N_{\beta}^{i}}{I_{\beta}^{i} \varepsilon_{\beta}^{i}}}=\frac{\bar{\varepsilon}_{\beta}}{\bar{\varepsilon}_{\beta}^{\prime} \bar{\varepsilon}_{n}} \frac{N_{\beta n}}{N_{\beta}}
$$

85 Here $N_{\beta n}^{i f}$ is the number of detected neutrons from the transition $i \rightarrow f$ in coincidence with betas populating level $i$. This ${ }_{140}$ requires detection of both the $\beta$ particle $\left(\varepsilon_{\beta}^{i}\right)$ and the neutron $\left(\varepsilon_{n}^{i f}\right)$. On the right-hand side of Eq. 2 the symbol $\bar{\varepsilon}_{\beta}^{\prime}$ represents the $\beta$ efficiency averaged over neutron unbound states, different from the $\beta$ efficiency averaged over all levels $\bar{\varepsilon}_{\beta}$.

Neglecting the energy dependency of the $\beta$ and neutron detection efficiencies can be an important source of systematic error. For instance the neutron moderation process in a counter of the type used here can vary appreciably with initial neutron energy leading to large efficiency variations. Since the neutron energy distribution is often unknown such detectors are sometimes designed to produce an extremely flat efficiency response [18]. However such designs reduce the average detection efficiency and therefore designs where the detection efficiency is maximized are also favoured. In the latter case the average neutron detection efficiency is more sensitive to the neutron energy spectrum given by $\sum_{i, E_{i}>S_{n}} \sum_{f} I_{\beta}^{i} I_{n}^{i f} E_{n}^{i f}$. The systematic correction to $\bar{\varepsilon}_{n}$ due to the neutron energy distribution for different $\beta$-delayed neutron emitters can be evaluated using Monte Carlo simulations as shown in Section 5.

Due to the continuum nature of the $\beta$ spectrum the low energy threshold in the electronic system introduces a strong energy dependence of the $\beta$ counting efficiencies for small $Q_{\beta}-E_{i}$ endpoint energies. This causes isotope dependent effects on the average $\beta$ detection efficiency and, more importantly, it can introduce large differences between $\bar{\varepsilon}_{\beta}^{\prime}$ and $\bar{\varepsilon}_{\beta}$. As a consequence the cancellation of the average $\beta$ efficiencies on the right-hand side of Eq. 2, a usual assumption, can lead to large errors. In Section 5 we evaluate the magnitude of such errors for the present setup using Monte Carlo simulations.

\section{Experimental apparatus}

\subsection{Neutron and beta counters}

The measurements were performed at the IGISOL mass separator [19] installed at the Cyclotron Laboratory of the University of Jyväskylä. A broad range of isotopes are produced in the proton-induced $\left(E_{p}=25 \mathrm{MeV}\right)$ fission of a thin thorium target inside the ion source. Reaction products which exit the target are swept away by a Helium gas jet into the $30 \mathrm{kV}$ electrostatic accelerating stage of the separator. The low energy ion beam is mass separated in a large dipole magnet with a modest mass resolution $M / \Delta M \sim 250$. In order to separate the nucleus of interest from accompanying isobars, the beam is directed to a double Penning trap system [20] working as a very high resolution mass separator. The purification cycle in the trap lasts for about $200 \mathrm{~ms}$ after which time the bunch of ions is released towards the experimental station. The extracted beam is isotopically pure to a high degree. Isobars from the mass separator are effectively suppressed in the trap, provided their mass difference with respect to the selected isotope is large in comparison to the trap frequency width. This was the case in our measurement. An exception is the production of nuclei inside the trap by decay of the selected isotope towards the end of the purification cycle. Beta-decay daughters will be doubly charged and have huge motional amplitudes in the trap resulting in a very small chance, estimated to be on the level of few percent or 
lower, to be extracted and implanted. This type of contamination could affect the measurement of isotopes with very short half-lives. The possible impact in our results is evaluated in Section 4.

The beam travels inside a $1 \mathrm{~mm}$ thick aluminium vacuum ${ }^{180}$ tube with a diameter of $46 \mathrm{~mm}$ and is implanted on a movable tape supported on a two roller system situated at a distance of about $2 \mathrm{~m}$ from the exit of the trap (see Fig. 1). The space between the rollers is $12 \mathrm{~mm}$. The tape used is a standard half-inch wide computer tape with the magnetic layer facing the beam. ${ }^{185}$ We estimate that the implantation depth of the ions is about a few tens of nanometers. At a distance of $6 \mathrm{~mm}$ behind the tape is situated a $0.5 \mathrm{~mm}$ thick $\mathrm{Si}$ detector with an active diameter of $25 \mathrm{~mm}$ mounted on a PCB frame. The use of such a thin $\mathrm{Si}$ detector minimizes $\gamma$ ray interactions. This detector has a geo-190 metrical efficiency of about $28 \%$ for counting $\beta$ particles emitted by the implanted ion. During the initial measurements we found that the direction of the beam extracted from the trap was drifting with time. The effect is amplified by the long distance and as a result the implantation position was changing enough ${ }^{195}$ to produce variations in the $\beta$ detection efficiency as large as a factor of two when comparing different runs. This effect would have been disastrous for the determination of $P_{n}$ values, therefore two collimators with holes having diameters of $10 \mathrm{~mm}$ and $5 \mathrm{~mm}$ were placed at convenient positions along the tube. In this way the change of $\beta$ efficiency during the whole beam time was reduced to a negligible value as will be shown in Section 5.

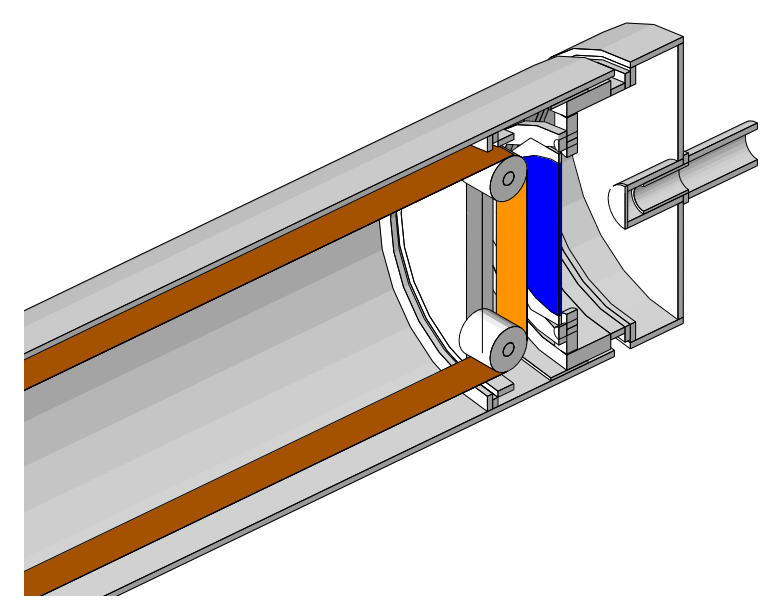

200

Figure 1: Cut-away view of the beam tube around the implantation point in the setup as implemented in the Geant 4 simulation code. In orange the implantation tape, in blue the Si detector.

The beam tube was placed inside the central hole of the neu-205 tron counter in such a way that the implantation position is at the center of the detector. The BELEN-20 version of the neutron counter used in this measurement consists of twenty ${ }^{3} \mathrm{He}$ proportional tubes arranged in two rings, with eight and twelve tubes respectively, around the central hole (see Fig. 2) The cen-210 tral hole has a radius of $55 \mathrm{~mm}$ and the detector rings have radii cal hole made in the polyethylene moderator with a diameter of $27.5 \mathrm{~mm}$. The proportional tubes were fabricated by LND Inc. [21] and have an external diameter of $25.4 \mathrm{~mm}$. The gas volume has an active length of $600 \mathrm{~mm}$. The total length of the tube including the HV connector is $676 \mathrm{~mm}$. The tube wall is made of stainless steel and has a thickness of $0.5 \mathrm{~mm}$. The tube is held in position inside the hole by means of a polyethylene plug with a hole for the high-voltage connection (see Fig. 2). The gas is a mixture of ${ }^{3} \mathrm{He}$ with $3 \%$ of $\mathrm{CO}_{2}$ at a pressure of 20 atmospheres. The neutron moderator block is made with slabs of high density polyethylene (PE) with a measured density of $\rho=0.955 \mathrm{~g} / \mathrm{cm}^{3}$. Seven slabs with a thickness of $100 \mathrm{~mm}$ and a cross section of $500 \times 500 \mathrm{~mm}^{2}$ make the core of the PE moderator. The eighth slab at the end acts as shielding against the external neutron background. The neutron shielding on the sides of the moderator block has a thickness of $200 \mathrm{~mm}$ and is assembled from twelve different PE slabs. The overall dimensions of the PE block are $900 \times 900 \times 800 \mathrm{~mm}^{2}$. The distribution of tubes inside the neutron moderator was obtained [22] as a result of MC simulations with MCNPX [23] and Geant4 [24]. This particular arrangement is optimized to enhance neutron detection efficiency.

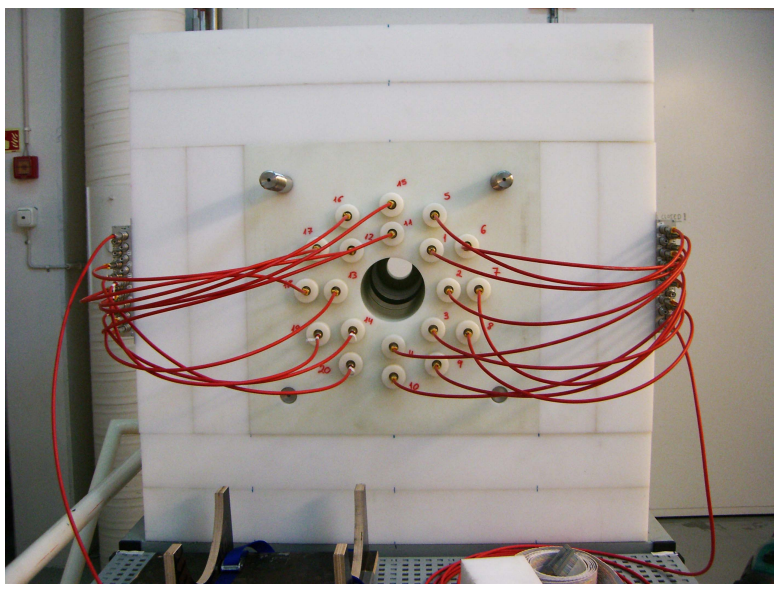

Figure 2: Front view of the BELEN-20 detector showing the disposition of the tubes around the central hole. The tubes are connected to the preamplifiers on both sides. The beam tube (not shown) is inserted from the back.

The pulses from the $\mathrm{Si}$ detector are integrated in a charge sensitive preamplifier model TC170 from Tennelec and shaped with an Ortec 671 spectroscopy amplifier. The signals from the ${ }^{3} \mathrm{He}$ tubes are fed to two MPR-16-HV charge sensitive preamplifiers from Mesytec. These 16 channel modules have a differential output and are connected to Mesytec 16 channel spectroscopy amplifiers model STM-16+. All preamplifier signals are shaped to Gaussian pulses with a shaping time of $1 \mu \mathrm{s}$. We found that the use of the shaper allowed us to have a better separation from the noise. A home-made fixed frequency clock $(10 \mathrm{~Hz})$ is used to trigger a tail pulse generator model $\mathrm{BH}-1$ from Berkeley Nucleonics Corporation. The pulser signal is sent to the test input of the preamplifiers and the height adjusted to have a peak at a convenient location in the amplitude spectra. The pulser allows the precise measurement of the real 
data acquisition time (live time).

\subsection{Self-triggered digital data acquisition system}

The time for neutrons to moderate their energy in the polyethylene and be captured in ${ }^{3} \mathrm{He}$ is quite long, up to several hundreds of microseconds (see Fig. 6 and Section 4). This affects the performance of conventional triggered data acquisi-275 tion systems (DACQ). The registration of decay events including both the neutron and the prompt detected radiation, $\beta$ particles or $\gamma$ rays, requires an event gate of similar magnitude and is thus affected by a large dead time. The long event gate also enhances the chance of registering uncorrelated signals and the 280 registration of multiple signals in the same channel. The separation of random and true coincidences is best done by studying all time correlations in the event time window. But in addition to the time it is important to measure the amplitude of every registered signal. This allows one, for instance, to discriminate 285 efficiently against fluctuating detector noise. However measurthe gate. All of these issues can be resolved with a self-triggered DACQ based on sampling digitizers where each DACQ detector channel runs independently [25]. Such a system has a very 290 much reduced intrinsic dead time. A potential problem with systems is the large amount of data which needs to be transferred (introducing additional dead time) and stored. The solution adopted for the BELEN DACQ is to use pulse self triggering and on-board processing of the digitized signal to obtain 295 for every pulse a time reference (time stamp) and the amplitude [26]. This reduces tremendously the amount of data to be transferred.

The DACQ used during the 2010 campaign is based on SIS3302 VME digitizers from Struck Innovative Systeme [27].300 These are 8 channel modules with $100 \mathrm{MHz}$ sampling freProgrammable Gate Array (FPGA) that stores the firmware to process the digitized pulse. We use the standard Gamma firmware from the manufacturer which matches our require- 305 ments for on-board data processing. A trapezoidal Finite Impulse Response (FIR) filter produces a short waveform for discrimination purposes (fast filter). Signals out of the fast filter that are larger than a given threshold generate an internal trigger for processing the input pulse with a second FIR filter for ${ }_{310}$ precise amplitude determination (slow filter). At the same time

255 the crossing of the threshold by the fast filter signal retrieves the content of a sample counter with 48 bit capacity which provides an absolute time stamp with $10 \mathrm{~ns}$ resolution. The slow filter is of trapezoidal type with compensation. The latter term ${ }_{315}$ refers to a correction for preamplifier signal fall time, which the 260 firmware presumes is the shape of the input signal. The parameters of both filters can be adjusted independently according to the characteristics of the input signal. The timing resolution of the fast filter applied to the shaped signals was very poor (over $_{320}$ $100 \mathrm{~ns}$ ) but it is of no relevance in the present application. The Section 3.1) produces some distortion of the amplitude spectrum (see lower panel in Fig. 3) which is also of no concern in the present application. The parameters of the slow filter that ${ }_{325}$ we use lead to a signal processing time of $10 \mu \mathrm{s}$. As every 70 channel is independent the rate dependent dead time has to be determined for every one. This is done with a fixed frequency pulser distributed to all channels via the preamplifiers as mentioned above.

Each acquisition channel has a 64 MByte on-board memory, where the result of the digital processing of the signal is stored. Typically we reduce the stored pulse information to the minimum, which includes the time stamp and the amplitude of the slow filter output. This requires 24 bytes of space per pulse. It also includes a flag signaling pulse pile-up when a second pulse within the processing time produces an internal trigger. Other event data storage options are available including the storage of the pulse waveform or the output of the slow filter. Each channel memory is divided into two banks. This allows one to accumulate data in one bank and at the same time retrieve the stored data from the second bank to the computer. This arrangement contributes to reducing the DACQ overall dead-time. The communication with the computer occurs via an optical link, which connects the SIS1100 PCI card with the SIS3100 VME interface card (both from Struck).

The gasificTL [28] data acquisition software is organized into four parallel processes which are responsible for: 1) hardware configuration and control, 2) block data read-out, 3) data storage on permanent media, and 4) on-line analysis. A fifth process, the Graphical User Interface (GUI), facilitates the control of these tasks. The GUI is built using Qt software [29]. For the communication and synchronization of the different processes we use Inter Process Communication (IPC) libraries available in POSIX [30]. Data is shared between processes though memory mapped files. The use of semaphores regulates the traffic of data and resolves conflicts between processes. Data storage on disk has priority over the other processes. Read-out from a data bank, and accumulation on the alternative bank, is started by a Look At Me (LAM) signal generated when any channel memory is almost full or after a predetermined time. On-line analysis can be very demanding, in particular the reconstruction of events and time correlations, and the software uses parallel processing to speed it up. Nevertheless typically only a fraction of the stored data is analyzed on-line. For construction, visualization and manipulation of histograms the DACQ relies on the ROOT data analysis framework [31]. The DACQ software uses a custom library which provides services as input/output abstraction, allowing to process data from hardware, filesystem or network indistinctly, management of DACQ configuration and setup, time stamp sorting, event windowing for classification and packaging, and data transformation for second level analysis. The library is written in $\mathrm{C}++$ using the standard template library which provides genericity, predictable behavior in memory management and a well know algorithm cost.

The latest version of the DACQ also uses the SIS3316, a 16 channel digitizer with 250 MSamples/s and 14 bit resolution from Struck. The firmware incorporates new features, including a Constant Fraction Discrimination (CFD) algorithm which provides improved time resolution. The new DACQ was used successfully in a recent measurement with BELEN48 at Jyväskylä. The new system has been upgraded to han- 
dle multiple VME crates, and a total of 192 acquisition channels. This extension is required to match the neutron detector of the BRIKEN project [17]. The DACQ has been also applied to other types of detectors like liquid scintillation detectors for neutron detection [32] and a $\mathrm{NaI}(\mathrm{Tl})$ total absorption $\gamma$-ray spectrometer [33].

\section{Measurements and data analysis}

To characterize the neutron-beta counting system we measured four well known $\beta$-delayed neutron emitters: ${ }^{88} \mathrm{Br},{ }^{94} \mathrm{Rb}$, ${ }^{95} \mathrm{Rb}$ and ${ }^{137} \mathrm{I}$. Table 1 gives their half-life $T_{1 / 2}$ taken from ENSDF [34, 35, 36, 37], total decay energy $Q_{\beta}$, and daughter neutron separation energy $S_{n}$ taken from [38] and the neutron emission probability $P_{n}$ taken from [39].

Table 1: Half-life $T_{1 / 2}$, decay energy window $Q_{\beta}$, daughter neutron separation energy $S_{n}$, and neutron emission probability $P_{n}$ for each measured isotope.

\begin{tabular}{ccccc}
\hline Isotope & $\begin{array}{c}T_{1 / 2} \\
(\mathrm{~s})\end{array}$ & $\begin{array}{c}Q_{\beta} \\
(\mathrm{MeV})\end{array}$ & $\begin{array}{c}S_{n} \\
(\mathrm{MeV})\end{array}$ & $\begin{array}{c}P_{n} \\
(\%)\end{array}$ \\
\hline${ }^{88} \mathrm{Br}$ & $16.34(8)$ & $8.975(4)$ & $7.053(3)$ & $6.75(18)$ \\
${ }^{94} \mathrm{Rb}$ & $2.702(5)$ & $10.283(3)$ & $6.831(8)$ & $10.24(21)$ \\
${ }^{95} \mathrm{Rb}$ & $0.3777(8)$ & $9.228(20)$ & $4.348(6)$ & $8.87(29)$ \\
${ }^{137} \mathrm{I}$ & $24.5(2)$ & $6.027(8)$ & $4.02556(10)$ & $7.33(38)$ \\
\hline
\end{tabular}

Each $\beta$-delayed neutron precursor was implanted for a period of time equivalent to three half-lives. The measuring time started $1 \mathrm{~s}$ before the accumulation period and lasted for a period of ten half-lives. At this point the activity on the tape was moved out, the time stamp scaler reset to zero and a new measurement cycle started. During the tape transport and accumulation "off" periods the primary beam is kept on target but the secondary beam is deflected to a beam dump located far away from the experimental setup.

The amplitudes of the signals from the Si detector and each ${ }^{3}$ He tube are calibrated in energy and histogramed as shown in Fig. 3. We use the position of the peak in the tube response and assign it a value of $764 \mathrm{keV}$, the energy released in the reaction ${ }^{3} \mathrm{He}(\mathrm{n}, \mathrm{p}){ }^{3} \mathrm{H}$, to calibrate the neutron spectrum. The calibration of the $\beta$ spectrum is made by comparison with Monte Carlo simulations of the energy deposited in the Si detector (see Section 5). The good separation of neutron signals from the noise can be seen in the lower panel of Fig. 3. We tag as neutron signals those which have an amplitude in the range of $130 \mathrm{keV}$ to $920 \mathrm{keV}$. The noise level in the Si detector allows us to set a low 375 energy threshold of $100 \mathrm{keV}$ to tag $\beta$ signals (see upper panel . For each neutron or $\beta$ event that fulfills the energy condition we histogramed its time stamp. In this way growth and decay curves of the activity are reconstructed (see Fig. 4 and Fig. 5). We also construct $\beta$-neutron time correlation his-з80 tograms from these events. For every $\beta$ event the time stamp difference with all neutron events in a time interval ranging from $-1 \mathrm{~ms}$ to $+1 \mathrm{~ms}$ is histogramed. Figure 6 shows an example.
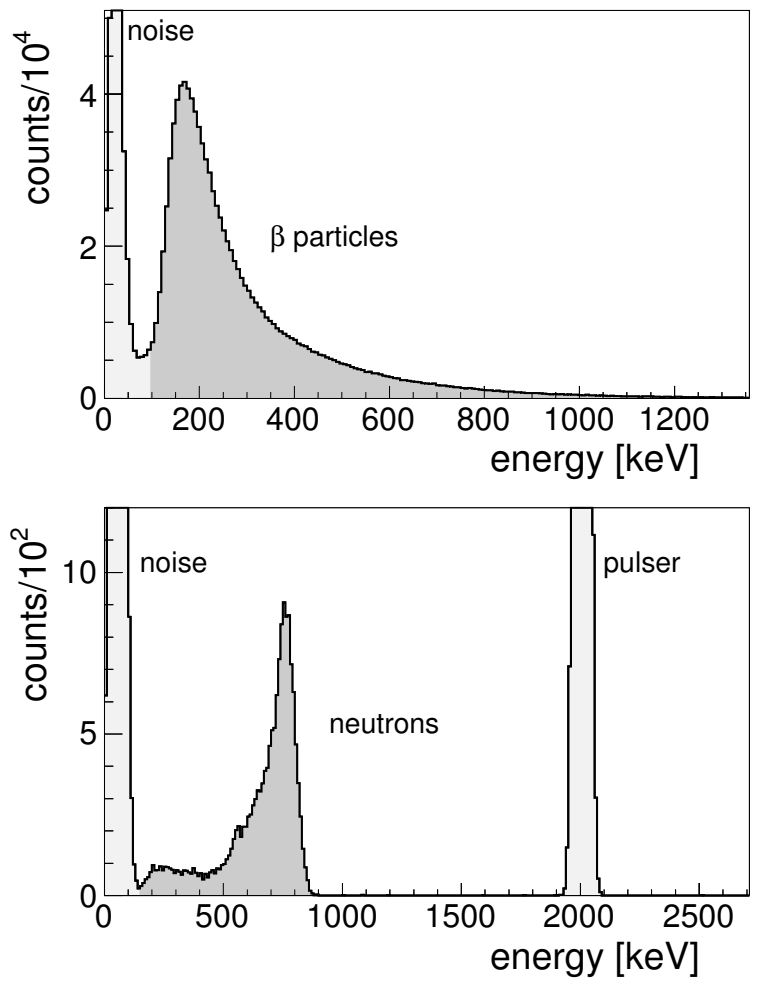

Figure 3: Upper panel: Energy spectrum recorded in the Si detector during the ${ }^{94} \mathrm{Rb}$ measurement. Lower panel: The energy spectrum recorded in the neutron detector during the measurement. The dark grey shaded area shows the events tagged as $\beta$ particles (upper panel) and neutrons (lower panel). Peaks due to noise and pulser signals are marked. In the Si detector the pulser peak is outside the range shown.

The asymmetric shape of the peak is due to the moderationplus-capture time distribution of neutrons in the detector. The mean value of this time distribution is approximately $80 \mu \mathrm{s}$ but the distribution extends up to about $500 \mu \mathrm{s}$.

The number of detected $\beta$ particles $N_{\beta}$ and neutrons $N_{n}$ which are to be ascribed to the decay of the parent nucleus is obtained from a time analysis decomposition of the growth and decay curves. This allows one to separate out the contribution from descendants. The time evolution of the number of counts due to all isotopes in the decay chain is described using the solution to the appropriate Bateman equations [40]. The experimental time distribution is fitted using either the chi-square minimization method or the maximum likelihood method for binned data. For that we use MINUIT optimization routines [41] which are integrated in ROOT. The fit function is based on the generic solution to the Bateman equations given in Ref. [42]. The solution has the form: 

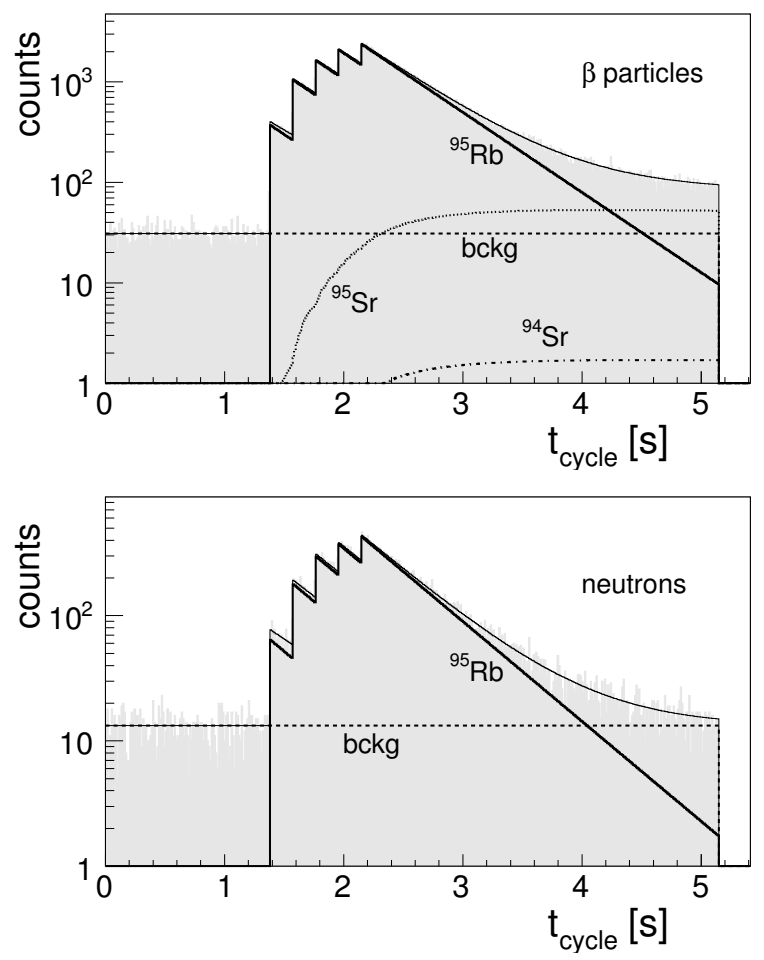

Figure 4: Decay of ${ }^{95} \mathrm{Rb}$. Upper panel: Fit to the distribution of $\beta$ counts as a function of cycle time. The different contributions to the fit are indicated: parent decay, decay of descendants and background. Lower panel: Fit to the distribution of neutron counts as a function of cycle time. The contribution to the fit of parent decay and background is shown.

$$
\begin{aligned}
N_{i}(t) & =\sum_{j=1}^{i} N_{j}\left(t_{0}\right)\left(\prod_{k=j}^{i-1}\left(\lambda_{k} b_{k, k+1}\right) \times \sum_{k=j}^{i} \frac{e^{-\lambda_{k}\left(t-t_{0}\right)}}{\prod_{l=j, l \neq k}^{i}\left(\lambda_{l}-\lambda_{k}\right)}\right) \\
& +\sum_{j=1}^{i} R_{j}\left(\prod_{k=j}^{i-1}\left(\lambda_{k} b_{k, k+1}\right) \times \sum_{k=j}^{i} \frac{\left(1-e^{-\lambda_{k}\left(t-t_{0}\right)}\right)}{\lambda_{k} \prod_{l=j, l \neq k}^{i}\left(\lambda_{l}-\lambda_{k}\right)}\right)
\end{aligned}
$$

$N_{i}(t)$ represents the number of $i$ nuclei at time $t, i$ representing the ordering number in the decay chain, $N_{i}\left(t_{0}\right)$ the number of nuclei at the initial time $t_{0}, R_{i}$ its constant rate of production, and $\lambda_{i}=\ln 2 / T_{1 / 2}^{i}$ its decay constant. The term $b_{k, k+1}$ represents the branching ratio between two successive isotopes in ${ }_{445}$ the decay chain. This formula is adjusted to the conditions of our measurement where only the parent nucleus was implanted. We use two forms, one assuming constant continuous implantation (see Fig. 5), and the other a series of instantaneous implantations (see Fig. 4). The latter is applied for the very short-450 lived isotopes where the pulsed nature of the beam from the trap except $R_{1}$. The function is defined in this way up to the end of the implantation period, determining the number of nuclei of each species formed up to this time which become $N_{i}\left(t_{i m p l}\right)$. Afterwards the time evolution is calculated setting $R_{i}=0$. In the discrete implantation case we set to zero all $R_{i}$ and all $N_{i}\left(t_{k}\right)$ at each implantation time $t_{k}$ except $N_{1}\left(t_{k}\right)$.

All decaying isotopes contributing to the time distribution are included in the fit. In the cases studied here, only the parent happens to be a $\beta$-delayed neutron emitter. Therefore the fit function for the $\beta$ time distribution includes two decay chains with $b_{12}$ weights, $1-P_{n}$ and $P_{n}$ respectively. The actual function has the form $\sum_{i} \bar{\varepsilon}_{\beta}^{i} \lambda_{i} N_{i}(t)$. In accordance with the discussion in Section 2 we use in this expression a different average $\beta$ efficiency for each isotope. However, as will be shown later, this dependency is very small in the present case and we can use the same efficiency $\bar{\varepsilon}_{\beta}$ for all of them. In the case of the neutron time distribution only the parent is included and the actual function is $\bar{\varepsilon}_{n} P_{n} \lambda_{1} N_{1}(t)$. A constant background is added to the fit function. The $1 \mathrm{~s}$ time period at the beginning of the measuring cycle, before implantation starts, serves to fix the background level. In the case of neutrons the background rate was $0.9 \mathrm{cps}$ during the measurements (accelerator on). For comparison, the rate descended to $0.7 \mathrm{cps}$ when the accelerator was turned off. The presence of a $\beta$ background, visible in all time spectra, is explained because the beam collimation system described above could not avoid that a fraction of the beam was implanted outside the tape, on the tape supporting structure or on the detector. We calculated the time dependence of the accumulated activity, not removed by the tape system, and concluded that after several measuring cycles it can be well represented by a constant value. For the nuclei analyzed here the values of $T_{1 / 2}$ for parent and descendants are well known. The same is true for the $P_{n}$ values. Therefore the only free parameter in the fit is the product of the number of implanted parent ions $\left(t_{\text {impl }} \times R_{1}\right.$ or $\left.\sum_{k} N_{1}\left(t_{k}\right)\right)$ times the detection efficiency. Integration of the parent activity curves provides $N_{\beta}$ and $N_{n}$. Figures 4 and 5 show examples of the fits obtained.

We have verified the assumption that only the parent nucleus is implanted. From the discussion in Section 3 and the half-live values in Table 1 , one can conclude that contamination with daughter nuclei from decay in the trap is more likely to occur in the case of ${ }^{95} \mathrm{Rb}$. Therefore we analyzed the time distribution of $\beta$ signals in the upper panel of Fig. 4 using a fit function which includes the implantation of daughter nuclei in addition to parent nuclei. A new fit parameter is introduced that corresponds to the fraction of daughter to parent nuclei. If this parameter is left free the best fit corresponds to values consistent with zero. If the parameter is fixed to $5 \%$ one observes a clear deterioration of the chi-square while the number of $\beta$ particles associated with the parent decay $N_{\beta}$ only changes by $0.4 \%$. Note that this fraction of daughter nuclei corresponds to the assumption that $10 \%$ of decay products produced in the trap are extracted an implanted, which is unrealistic. We conclude that for all practical purposes the beam is pure.

The case of ${ }^{137} \mathrm{I}$ (Fig. 5) and ${ }^{88} \mathrm{Br}$ (not shown) were special. In both cases the $\beta$ activity towards the end of the cycle was smaller than the calculated values. The solution to the puzzle 

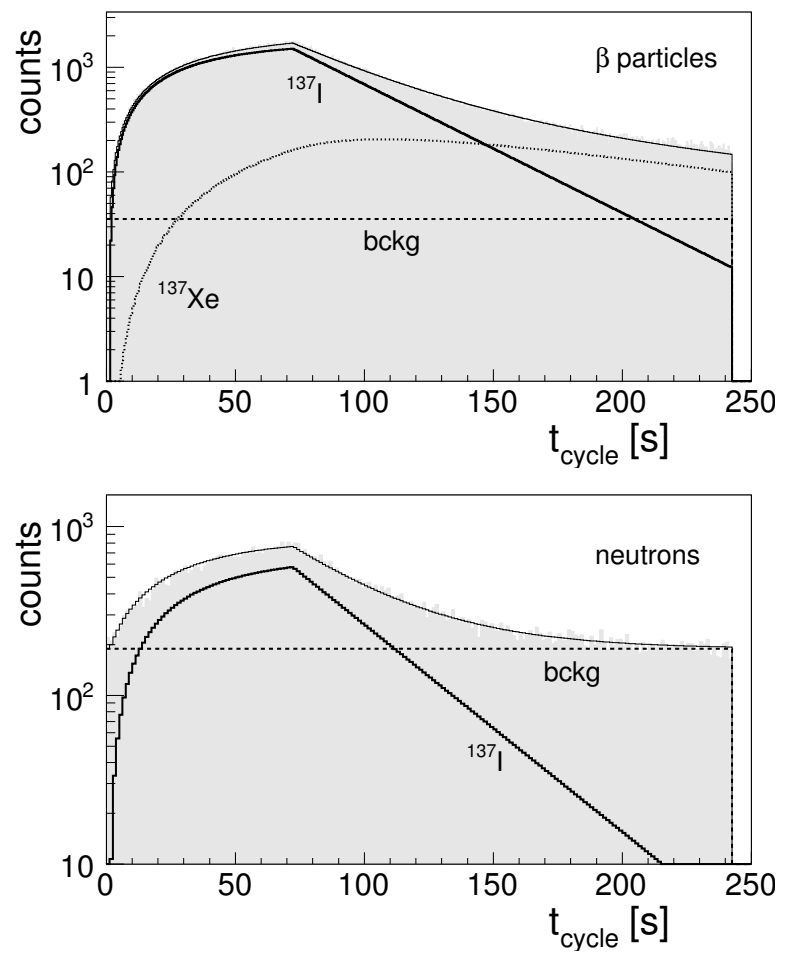

Figure 5: Same as Fig. 4 for the decay of ${ }^{137} \mathrm{I}$.

came with the realization that both descendants ${ }^{137} \mathrm{Xe}$ and ${ }^{88} \mathrm{Kr}$ are noble gases with a tendency to diffuse easily from organic plastic materials. The magnetic substrate of the implantation tape is actually composed of magnetic granules embedded in an acrylic layer. Therefore a fraction of daughter nuclei can escape from the tape and do not contribute to the measured $\beta$ rate. A similar effect has been observed before [43]. To solve the problem we added a loss term for $\mathrm{Xe}$ and $\mathrm{Kr}$ isotopes to the Bateman equations. The loss rate was assumed constant and the solution function was modified accordingly. The loss rate is an additional fit parameter in these cases. This adds a systematic uncertainty to the parent $\beta$ rate determined from the fit but we estimate that this uncertainty is small (see below).

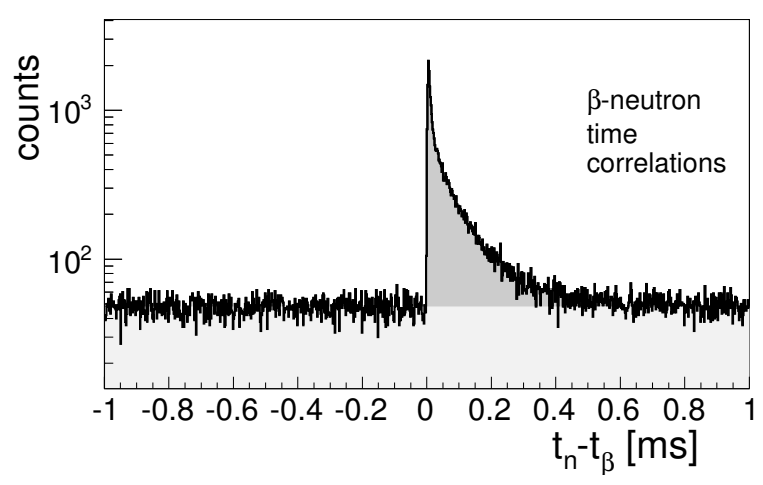

Figure 6: Decay of ${ }^{94} \mathrm{Rb}$. Time difference between all neutron events and a $\beta$ event in the range from $-1 \mathrm{~ms}$ to $+1 \mathrm{~ms}$.
To determine the number of $\beta$-neutron correlated events $N_{\beta n}$ we use the time correlation histograms (see Fig. 6). The negative time part of the histogram (backwards in time) represents faithfully the background of random correlated events under the true correlations in the forward time direction. As can be seen the rate of random events is constant in this time window. Therefore a fit to the negative part of the histogram is used to subtract the background from the positive time events. The length of the time window, $1 \mathrm{~ms}$, is long enough to ensure that all the neutrons are collected.

We give in Table 2 the values of $N_{\beta}, N_{n}$ and $N_{\beta n}$ obtained in the way described above for the four isotopes. These numbers have been corrected for data acquisition dead time, although the correction is very small, less than $0.3 \%$ in all cases. The dead time correction for each acquisition channel was determined by comparison of the number of counts in the peak due to the fixedfrequency pulse generator and the total measuring time. This was calculated from the number of measuring cycles and the cycle time length, determined from the cycle time histograms (such as those shown in Fig. 5).

Table 2: Number of $\beta$ counts $N_{\beta}$, neutron counts $N_{n}$, and $\beta$-neutron correlated counts $N_{\beta n}$ obtained for each measured isotope.

\begin{tabular}{cccc}
\hline Isotope & $N_{\beta}$ & $N_{n}$ & $N_{\beta n}$ \\
\hline${ }^{88} \mathrm{Br}$ & $1.0592(11) \times 10^{6}$ & $1.396(4) \times 10^{5}$ & $3.08(4) \times 10^{4}$ \\
${ }^{94} \mathrm{Rb}$ & $6.915(8) \times 10^{5}$ & $1.388(4) \times 10^{5}$ & $3.42(4) \times 10^{4}$ \\
${ }^{95} \mathrm{Rb}$ & $1.793(4) \times 10^{5}$ & $3.219(18) \times 10^{4}$ & $8.05(22) \times 10^{3}$ \\
${ }^{137} \mathrm{I}$ & $2.464(5) \times 10^{5}$ & $3.76(3) \times 10^{4}$ & $7.14(10) \times 10^{3}$ \\
\hline
\end{tabular}

\section{Results and discussion}

From the numbers given in Table 2 and the known $P_{n}$ value (Table 1 ) we can calculate the quantity $\bar{\varepsilon}_{\beta} / \bar{\varepsilon}_{n}$ using Eq. 1 . This purely experimental quantity should be independent of the nucleus concerned except for the systematic effects discussed in Section 1. It characterizes the $\beta$-neutron counting setup and, once determined, allows one to obtain $P_{n}$ values for other isotopes. Figure 7 represents the ratio of average $\beta$ and neutron efficiencies for the four isotopes. The dashed line connects the uncorrected values and the solid line connects the values corrected by systematic effects on $\bar{\varepsilon}_{\beta}$ and $\bar{\varepsilon}_{n}$ as will be detailed below. As can be appreciated the corrections are very small. There is a very good agreement between the different isotopes which indicates that systematic errors are well under control, in particular the variations of $\beta$ efficiency with time. The weighted average of the ratio is $\bar{\varepsilon}_{\beta} / \bar{\varepsilon}_{n}=0.506(7)$, which has an uncertainty of only $1.4 \%$. This value has been used to determine the $P_{n}$ values for other isotopes [14].

To compute the corrections to the neutron efficiency coming from the neutron energy distribution we have used Geant4 MC simulations. We implemented a particle generator which reproduces the neutron energy distribution taken from the ENDF/BVII.1 [44] nuclear data base. The data in this file come from the 


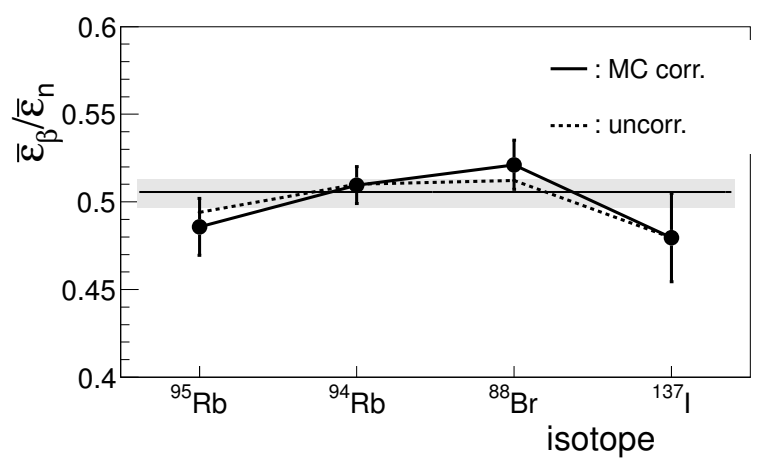

Figure 7: Ratio of average $\beta$ to average neutron efficiencies for the four measured isotopes. The solid horizontal line represents the weighted average and the shaded area its uncertainty.

evaluation work of Ref. [45] and are supplemented with theoretical calculations [46] outside the measured energy range. Figure 8 shows the neutron energy distributions for ${ }^{88} \mathrm{Br}$ and ${ }^{137} \mathrm{I} .{ }^{94} \mathrm{Rb}$ and ${ }^{95} \mathrm{Rb}$ are excluded for clarity. The figure shows also the neutron detection efficiency as a function of neutron energy obtained from the Geant 4 simulations. The simulations were performed with version 10.0 (patch 3) of the simulation toolkit. In the code we include a detailed geometrical description of the BELEN neutron counter. As can be observed the efficiency is rather constant below $0.5 \mathrm{MeV}$, with a value of about ergy, being only $29 \%$ at $5 \mathrm{MeV}$. Although the energy window for neutron emission $Q_{\beta n}=Q_{\beta}-S_{n}$ varies from $1.9 \mathrm{MeV}$ for ${ }^{88} \mathrm{Br}$ to $4.9 \mathrm{MeV}$ for ${ }^{95} \mathrm{Rb}$ (see Table 3 ), the Fermi decay rate function shifts the neutron spectrum to rather low energies as . In fact the largest average neutron energy $\left\langle E_{n}\right\rangle$ is $625 \mathrm{keV}$ and corresponds to the decay of ${ }^{137} \mathrm{I}$. Because of this one expects a modest isotope dependency for the average neutron efficiency. This is confirmed by the simulation as shown in Table 3.

${ }_{530}$ In Table 3 and in Fig. 8 we also show data for the ${ }^{252} \mathrm{Cf}$ spontaneous-fission neutron source. This source is often used to calibrate neutron detectors. The Californium neutron energy spectrum shown in Fig. 8 is taken from Ref. [47]. The spectrum reaches $25 \mathrm{MeV}$ and has an average energy of $2.2 \mathrm{MeV}$. Thus it senses a portion of the efficiency curve different from the fission products. The result of the simulation gives an average neutron efficiency of $39.5 \%$. We used a calibrated ${ }^{252} \mathrm{Cf}$ source to measure the neutron detection efficiency and obtained a value of $40.9(8) \%$ in good agreement with the simulation. It should be noted that this level of agreement could only be reached after correction of some bugs [48] in Geant4 which have been incorporated in version 10.0 and later versions of the code. The ratio between the counts of the inner and the outer ring of ${ }^{3} \mathrm{He}$ tubes is quite sensitive to the neutron energy distribution [10]. The measured ratio is $1.499(3)$ in quite good $_{570}$ agreement with the result of the simulation 1.52. In addition it is worth mentioning that the simulation reproduces well the neutron moderation-plus-capture time distribution, like the one shown in Fig. 6. These results show the suitability of Geant4 mulating the response of this type of neutron detector

The correction factor to be applied to $\bar{\varepsilon}_{n}$ for each isotope is calculated as the ratio of the simulated efficiency in Table 3 to the average value for the four isotopes. The correction is small, varying between $-1.1 \%$ for ${ }^{95} \mathrm{Rb}$ and $+1.2 \%$ for ${ }^{88} \mathrm{Br}$. Hower the correction can be important for decays where the $Q_{\beta n}$ window is large and the $\beta$ intensity distribution is sizable at excitation energies well above $S_{n}$. Such a situation can be found in lighter nuclei. It is also possible that nuclear structure effects could produce a similar situation for other nuclei with very low level density, such as those close to doubly magic nuclei. It is obvious that this way of calculating the corrections to the average neutron efficiency can only be applied if the $\beta$-delayed neutron energy spectrum is known. When this is not the case the magnitude of the systematic error due to the unknown neutron energy distribution can be obtained from assumptions or calculations of the $\beta$ intensity distribution. For detectors having more than two rings of tubes, an improved estimate of the average neutron efficiency can be obtained using the information from the ratio of counts between different rings [10].

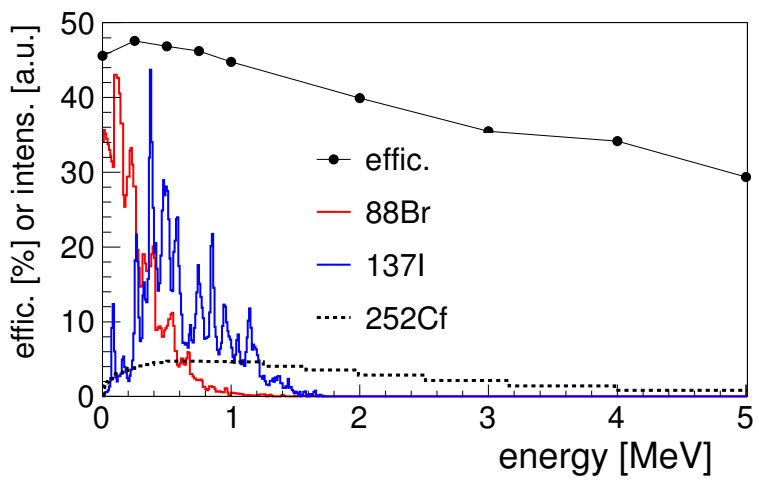

Figure 8: BELEN-20 neutron detection efficiency as a function of neutron energy simulated with Geant4. Superimposed is the neutron energy spectra for ${ }^{88} \mathrm{Br}$ and ${ }^{137} \mathrm{I}$ fission products, and the ${ }^{252} \mathrm{Cf}$ calibration neutron source. The spectra are scaled with a common arbitrary factor.

Table 3: Energy window for neutron emission $Q_{\beta n}$, average neutron energy $\left\langle E_{n}\right\rangle$ and Geant4 simulated average neutron detection efficiency $\bar{\varepsilon}_{n}$ for each measured fission product and the ${ }^{252} \mathrm{Cf}$ calibration neutron source.

\begin{tabular}{cccc}
\hline Isotope & $\begin{array}{c}Q_{\beta n} \\
(\mathrm{MeV})\end{array}$ & $\begin{array}{c}\left\langle E_{n}\right\rangle \\
(\mathrm{MeV})\end{array}$ & $\begin{array}{c}\bar{\varepsilon}_{n} \\
(\%)\end{array}$ \\
\hline${ }^{88} \mathrm{Br}$ & 1.922 & 0.247 & 47.0 \\
${ }^{94} \mathrm{Rb}$ & 3.452 & 0.436 & 46.7 \\
${ }^{95} \mathrm{Rb}$ & 4.880 & 0.525 & 46.0 \\
${ }^{137} \mathrm{I}$ & 2.001 & 0.625 & 46.2 \\
${ }^{252} \mathrm{Cf}$ & - & 2.2 & 39.5 \\
\hline
\end{tabular}

We now consider the corrections to the $\beta$ efficiency coming from the $\beta$ intensity distribution. In Fig. 9 we show the $\beta$ efficiency as a function of end point energy obtained from Geant 4 simulations. The simulation application includes a detailed description of the geometry of the implantation setup (see Fig. 1): 

vacuum tube and end-cap. For the efficiency calculation we assume a $\beta$ spectrum shape of allowed type. The use of forbidden shapes has a minor impact on the efficiency. The simulated ${ }_{635}$ efficiency is scaled down with a factor of 0.806 to take into acthat part of the beam is implanted outside the tape and is not seen by the Si detector. How this factor was determined is explained below. As can be observed the efficiency varies strongly up to $2 \mathrm{MeV}$ due to the effect of the $100 \mathrm{keV}$ detection 640 threshold in the Si detector. In the figure we also show the $\beta$ intions are scaled for clarity. The lower panel shows the intensity $I_{\beta \gamma}$ that is followed by $\gamma$ emission or feeds the ground state in the daughter nucleus. For ${ }^{95} \mathrm{Rb}$ and ${ }^{137} \mathrm{I}$ the distribution is taken ${ }_{645}$ from the ENSDF data base $[36,37]$ and was obtained with highresolution $\gamma$-ray spectroscopy using germanium detectors. For ${ }^{88} \mathrm{Br}$ and ${ }^{94} \mathrm{Rb}$ we show the result of recent experiments [49] using total absorption $\gamma$-ray spectroscopy (TAGS). The upper panel shows the $\beta$ intensity distribution that is followed by neu-650 tron emission $I_{\beta n}$. This intensity distribution was obtained from the deconvolution of the neutron spectra shown in Fig. 8. The total $\beta$ intensity $I_{\beta}$ is obtained from summation of the two distributions $I_{\beta \gamma}$ and $I_{\beta n}$ with the proper normalization, $1-P_{n}$ and $P_{n}$ respectively. This intensity distribution can be used as input for a Geant 4 simulation to determine the average $\beta$ efficiency $\bar{\varepsilon}_{\beta}$ which is shown in Table 4. As can be observed the dependency on the nucleus concerned is very small, a maximum variation of half-a-percent with respect to the average. This was expected from the large values of the average end-point energy for the decay $Q_{\beta}-\left\langle E_{x}\right\rangle_{I_{\beta}}$ also shown in Table 4 .

A comment on the accuracy of $\beta$ intensity distributions is pertinent at this point. Intensity distributions coming from highresolution spectroscopy are often affected by systematic errors, as a consequence of the limited efficiency of germanium detectors. Gamma rays de-exciting high energy levels can be easily missed or cannot be placed in the level scheme. This leads to an incomplete and distorted level scheme with too much intensity assigned to levels at low excitation energy. This can be clearly seen in the case of the decay of ${ }^{94} \mathrm{Rb}$ where the ENSDF data evaluation [35] locates only $66 \%$ of $I_{\beta \gamma}$. Total absorption spectroscopy with large $4 \pi$ scintillation detectors gives the correct intensity distribution. Indeed the TAGS result for ${ }^{94} \mathrm{Rb}$ [49] places considerable intensity at high-excitation energy. In spite of that the calculated average $\beta$ efficiency does not change significantly.

620 Summarizing, the corrections due to the energy distribution of neutrons and $\beta$ particles on the ratio $\bar{\varepsilon}_{\beta} / \bar{\varepsilon}_{n}$ for the measured isotopes are very small and do not add much to the systematic uncertainty of the result.

From the four isotopes, ${ }^{137} \mathrm{I}$ is the one with the largest relative uncertainty on the $P_{n}$ value. According to the evaluation of Ref. [39] the uncertainty amounts to 5.2\% (see Table 1 and Fig. 7). We can use the $P_{n}$ values for the other three isotopes to obtain an improved estimate of the $\beta$-delayed neutron emission probability for this important $\beta$-delayed neutron precursor. ${ }^{137} \mathrm{I}$ is one of the single largest contributors to the delayed neutron fraction in a reactor. The ratio $\bar{\varepsilon}_{\beta} / \bar{\varepsilon}_{n}$ determined with the ex-655 clusion of ${ }^{137} \mathrm{I}$ is $0.508(8)$, only marginally different from the number given above. With this ratio we determine a more accurate neutron emission probability $P_{n}=7.76(14) \%$ for ${ }^{137} \mathrm{I}$. As was explained in Section 4 the measurement for this isotope is affected by the escape of the daughter nuclei from the implantation tape. This introduces a systematic uncertainty on the number of $\beta$ counts $N_{\beta}$ related to the modeling of this effect in the fit function. However this uncertainty is very small. ${ }^{137} \mathrm{Xe}$ decay is the only significant additional contribution to the growth and decay curve (see Fig. 5). Since the half-life of the daughter is nine times longer than the parent half-life, the contribution is small during the first part of the measuring cycle. Restricting the fit of $\beta$ counts in Fig. 5 up to the end of the implantation period $(73 \mathrm{~s})$, and removing the loss term from the fit function, we obtain a number of counts in the full cycle $N_{\beta}$ which differs only by $0.4 \%$ from the value given in Table 2 . We assume that this difference gives the magnitude of the xenon loss systematic error and include it in the quoted uncertainty given above. Our new determination of ${ }^{137} \mathrm{I} P_{n}$ value has thus an uncertainty of $1.8 \%$.
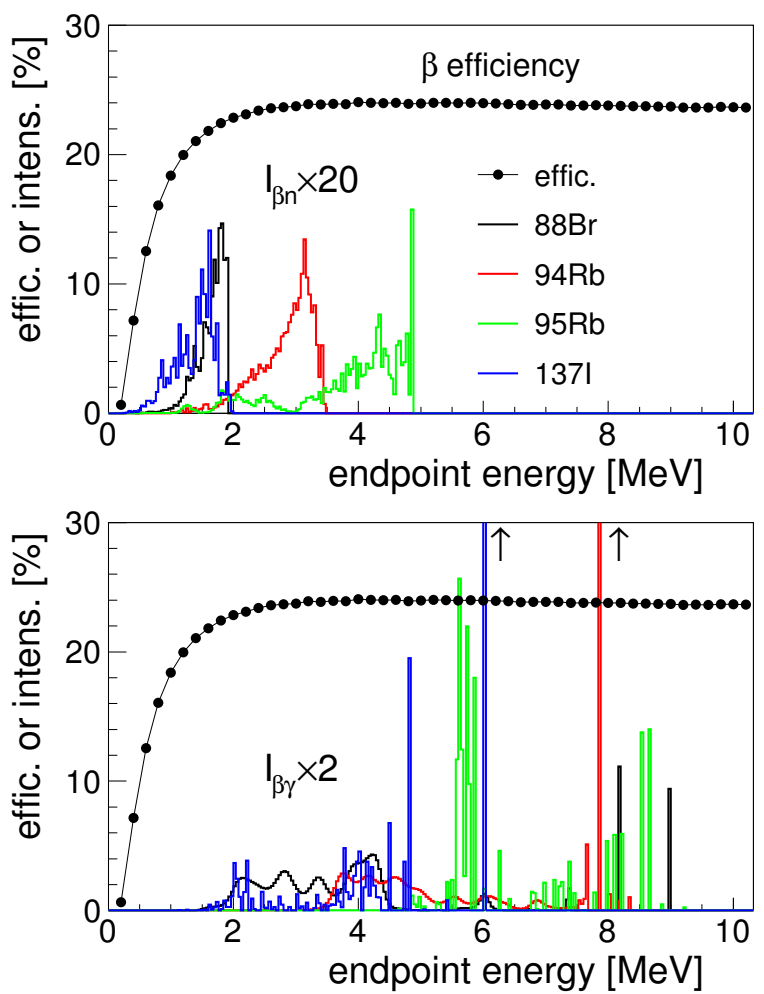

Figure 9: Beta detection efficiency of the Si detector as a function of $\beta$ endpoint energy obtained from Geant 4 simulations (solid circles). Lower panel: Superimposed is the portion of the $\beta$-intensity distribution followed by $\gamma$ emission. Upper panel: Superimposed is the portion of the $\beta$-intensity distribution followed by neutron emission. The intensity distributions are scaled with a common factor shown in the figure.

We turn now to consider the effect of the $\beta$ particle energy distribution on the determination of $P_{n}$ values using $\beta$-neutron coincidences Eq. 2. From observation of the upper panel of Fig. 9, which shows the $\beta$ intensity distribution followed by 
Table 4: Average $\beta$ end-point energy $Q_{\beta n}-\left\langle E_{x}\right\rangle_{I_{\beta}}$, average Geant4 simulated $\beta$ efficiency $\bar{\varepsilon}_{\beta}$, average $\beta$ end-point energy in the neutron emission window $Q_{\beta}-\left\langle E_{x}\right\rangle_{I_{\beta n}}$, and Geant 4 simulated average $\beta$ efficiency for decays to neutron unbound states $\bar{\varepsilon}_{\beta}^{\prime}$, for each measured fission product.

\begin{tabular}{ccccc}
\hline Isotope & $\begin{array}{c}Q_{\beta}-\left\langle E_{x}\right\rangle_{I_{\beta}} \\
(\mathrm{MeV})\end{array}$ & $\begin{array}{c}\bar{\varepsilon}_{\beta} \\
(\%)\end{array}$ & $\begin{array}{c}Q_{\beta}-\left\langle E_{x}\right\rangle_{I_{\beta n}} \\
(\mathrm{MeV})\end{array}$ & $\begin{array}{c}\bar{\varepsilon}_{\beta}^{\prime} \\
(\%)\end{array}$ \\
\hline${ }^{88} \mathrm{Br}$ & 3.875 & 23.6 & 1.649 & 21.9 \\
${ }^{94} \mathrm{Rb}$ & 5.455 & 23.9 & 2.826 & 23.8 \\
${ }^{95} \mathrm{Rb}$ & 6.294 & 23.9 & 3.857 & 24.0 \\
${ }^{137} \mathrm{I}$ & 4.610 & 23.6 & 1.376 & 19.9 \\
\hline
\end{tabular}

neutron emission, one expects a larger influence than in the case of independent $\beta$ and neutron counting. These distributions sample with a large weight the portion of the $\beta$ efficiency curve which varies strongly because of the low energy threshold. Figure 10 shows the average $\beta$ efficiency $\bar{\varepsilon}_{\beta}^{\prime}$ determined experimentally as the ratio $N_{\beta n} / N_{n}$ (compare Equations 1 and $2)$. In the figure the isotopes are plotted in the order of decreasing average $\beta$ end-point energy $Q_{\beta}-\left\langle E_{x}\right\rangle_{I_{\beta n}}$, to make the trend clearer. The experimental value of the efficiency for detecting a $\beta$ particle in coincidence with the emitted neutron is $25 \%$ smaller for ${ }^{137} \mathrm{I}$ than for ${ }^{94} \mathrm{Rb}$ or ${ }^{95} \mathrm{Rb}$. This would have been the magnitude of the systematic error in the coincidence method for ${ }^{137}$ I if this effect was ignored.

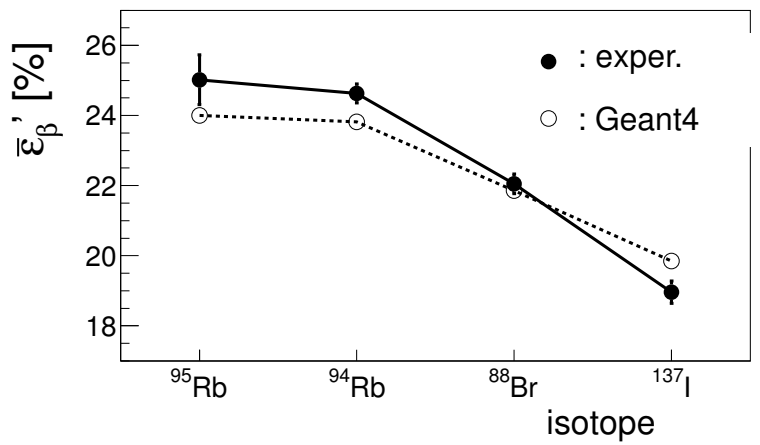

Figure 10: Detection efficiency of the Si detector for $\beta$ particles in coincidence with neutrons detected in BELEN for the four fission products. Closed symbols: measurement; open symbols: Geant 4 simulation.

The experimental determination of $\bar{\varepsilon}_{\beta}^{\prime}$ gives us the oppor-705 Geant4 simulations in the region of strong variation. This is important not only for estimating the corrections in the coincidence method of $P_{n}$ determination but in other types of decay measurements, that also require $\beta$ tagging. In particular the ${ }_{710}$ ticle eliminates the huge background in the large scintillation detector thus facilitating the measurement of rare isotopes. Determining accurately the $\beta$ intensity distribution close to the $Q$ value depends critically on our knowledge of the $\beta$ efficiency ${ }_{715}$ curve. In order to compare with the measurement we have generated $\beta$ events from the $I_{\beta n}$ distribution assuming an allowed $\beta$ shape. Figure 11 compares the experimental and simulated energy spectrum registered in the Si detector. It is remarkable that the simulation is able to reproduce the shapes, distinct for each isotope, which are sensitive to the details of the $\beta$ intensity distributions. The integral values are compared in Fig. 10. The MC simulation has been scaled down by a factor of 0.806 to match, on average, the experimental values. The simulation was performed assuming that the implantation position was at the centre of the tape. As was mentioned above, part of the beam was deposited on the supporting structure, which stopped the $\beta$ particles, thus the effective efficiency was $20 \%$ smaller than the nominal value. Apart from this geometrical factor the simulation reproduces the tendency of the measured values to better than $4.5 \%$. The scaled values are shown in Table 4.
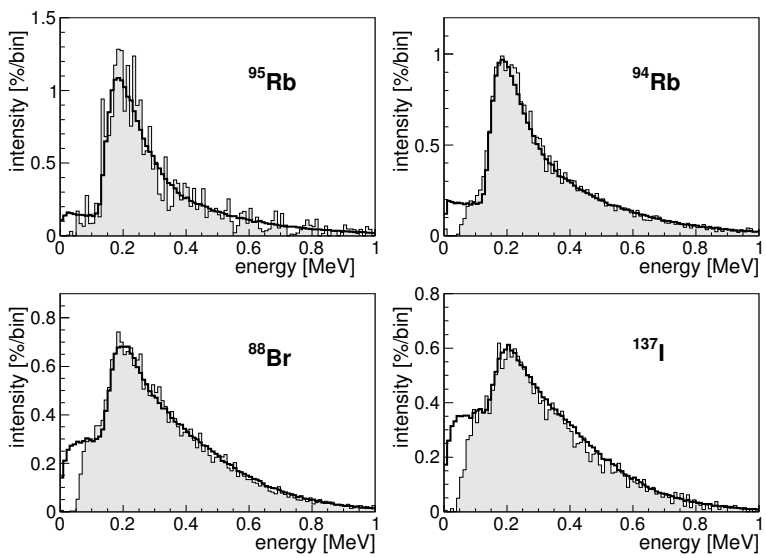

Figure 11: Energy spectrum measured in the Si detector in coincidence with neutrons detected in BELEN for the four measured delayed neutron precursors. Shaded histogram: measurement. Solid line: Geant4 simulation.

\section{Summary and conclusions}

We have described a new $\beta$-neutron counting system for the measurement of $\beta$-delayed neutron emission probabilities. The setup is adapted for measurements at on-line mass separators. The neutron counter uses twenty ${ }^{3} \mathrm{He}$ tubes distributed inside a large polyethylene neutron moderator. A thin Si detector is used as $\beta$ counter. A novelty of the apparatus is the use of a self-triggered digital data acquisition system with small acquisition dead time. The time and energy for every detector signal are stored for subsequent analysis. This allows a flexible reconstruction of events and full control of experimental issues in the data. The characterization of the counting system was performed using fission products with well known $\beta$-delayed neutron emission properties: ${ }^{88} \mathrm{Br},{ }^{94} \mathrm{Rb},{ }^{95} \mathrm{Rb}$ and ${ }^{137} \mathrm{I}$. The ratio of $\beta$ to neutron detection efficiencies for this setup, which is the parameter used for the determination of $P_{n}$ values, was determined with an uncertainty of $1.6 \%$. This allowed us to improve the $P_{n}$ for the important $\beta$-delayed neutron precursor ${ }^{137} \mathrm{I}$. Our new value is $(7.76 \pm 0.14) \%$.

We studied the effect of $\beta$ and neutron energy distributions on the average detection efficiencies. They can introduce a systematic error in the determination of $P_{n}$ values. We used Geant 4 
MC simulations to quantify the effect. For the method of independent $\beta$ and neutron counting we found that the corrections are very small for the four isotopes investigated. To a large ex- ${ }^{775}$ tent this is related to the strong energy dependence of the Fermi rate function which leads to large average $\beta$ end-point energies and small average neutron energies. In the general case, the corrections can be important for decays where the $\beta$ intensity ${ }^{70}$ distribution concentrates at very high excitation energies, due to nuclear structure, and/or onto few levels, as can happen for light nuclei or close to shell closures. The situation is quite different for the $\beta$-neutron coincidence counting method. The inevitable noise discrimination threshold in the $\beta$ counter leads to a strong efficiency variation with end-point energy in the upper part of the decay window. Thus the average efficiency for detecting a $\beta$ particle in coincidence with a neutron can be very different from the average efficiency for detecting any $\beta$ particle. The effect is exacerbated when the window for neutron emission decreases. We observed a $25 \%$ difference between ${ }^{137} \mathrm{I}$ and ${ }^{94} \mathrm{Rb}$ or ${ }^{95} \mathrm{Rb}$. This would have been the magnitude of the systematic error if this effect is ignored. On the other hand we found that Geant4 simulations are able to reproduce the isotope dependency of the neutron-gated $\beta$ efficiency within $4.5 \%$. Likewise , with the corrected thermal neutron treatment in version 10.0 and later versions, was able to reproduce the neutron detection efficiency of a calibrated ${ }^{252} \mathrm{Cf}$ source within $3.5 \%$. This confirms the suitability of Geant 4 to quantify systematic corrections to the $P_{n}$ value coming from the dependency of efficiency with energy. However to evaluate these corrections one needs information on the $\beta$ intensity distribution and the neutron energy spectrum. For the cases where this information is missing an estimate of the corrections can be obtained from theoretical calculations of the intensity distribution or from some reasonable assumption about this distribution.

\section{Acknowledgment}

This work has been supported by the Spanish Ministerio de Economía y Competitividad under grants FPA2008-06419,820 FPA2011-24553, PRI-PIMNUP-2011-1348 FPA2011-28770C03-03, and FPA2014-52823-C2. Supported by STFC (UK). Supported by the Academy of Finland under the Finnish Centre of Excellence Programme 2006-2011 (Nuclear and Accelerator Based Physics Programme at JYFL). Work partially done within IAEA-CRP for Beta Delayed Neutron Data.

\section{References}

[1] I.N. Borzov, Phys. Rev. C 71 (2005) 065801

[2] A. Arcones and G. Martinez-Pinedo, Phys. Rev. C 83 (2011) 045809.

[3] B. Rubio, Int. J. Modern Phys. E 15 (2006) 1979.

[4] A. Herlert, 2nd International Conference on New Frontiers in Physics, Crete (Greece), EPJ Web of Conferences 71 (2014) 00064.

[5] G. Rosner, Nucl. Phys. B Proc. Supp. 167 (2007) 77.

[6] F. Calviño et al., Technical Design Report for BELEN detector, NUSTAR-FAIR, 2014. https://greener.upc.edu/greter/BELEN/TDR_BELEN.pdf

$770 \quad$ [7] E. Roeckl et al., Phys. Rev. C 10 (1974) 1181.

[8] K.-L. Kratz et al., Nucl. Phys. A 229 (1974) 179

[9] G. Rudstam et al., Phys. Rev. C 13 (1976) 321.
[10] P.L. Reeder et al., Phys. Rev. C 15 (1977) 2098.

[11] J. Pereira et al., Nucl. Instrum Meth. A 618 (2010) 275.

[12] R. Grzywacz et al., Acta Physica Polonica 45 (2014) 217.

[13] M.B. Gomez-Hornillos et al., Hyperfine Interactions 223 (2014) 185.

[14] J. Agramunt et al., Nucl. Data Sheets 120 (2014) 74.

[15] R. Caballero-Folch et al., Nucl. Data Sheets 120 (2014) 81.

[16] A. D'Angelo, Prog. Nucl. Energy 41 (2002) 5.

[17] https://www.wiki.ed.ac.uk/display/BRIKEN/

[18] L. Mathieu et al., J. Instrum. 7 (2012) P08029.

[19] J. Äystö, Nucl. Phys. A 693 (2001) 477.

[20] T. Eronen et al., Eur. Phys. J. A 48 (2012) 46.

[21] LND, INC. http://www.lndinc.com/

[22] M.B. Gomez-Hornillos et al., J. Korean Phys. Soc. 59 (2011) 1573

[23] D. Pelowitz (Ed.), MCNPX Users Manual Version 2.5.0, Los Alamos National Laboratory, Report LA-CP-05-0369, April 2005.

[24] S. Agostinelli et al., Nucl. Instrum. Methods A 506 (2003) 250.

[25] U. Abbondanno et al., Nucl. Instrum. Methods A 538 (2005) 702

[26] W. Królas et al., Phys. Rev. C 65 (2002) 031303.

[27] Struck Innovative Systeme. http://www.struck.de/

[28] J. Agramunt et al., La Rabida 2012 International Meeting on Nuclear Physics, AIP Conf. Proc. 1541 (2013) 165.

[29] The Qt company. https://www.qt.io/

[30] http://standards.ieee.org/develop/wg/POSIX.html

[31] R. Brum and F. Rademakers, Nucl. Instrum. Methods A 389 (1997) 81.

[32] V. Modamio et al., Nucl. Instrum. Methods A 775 (2015) 71.

[33] V. Guadilla et al., International Conference on Electromagnetic Isotope Separators and Related Topics, EMIS-2015, Grand Rapids, May 11-15, 2015, submitted to Nucl. Instrum. Methods B.

[34] E.A. Mccutchan et al., Nucl. Data Sheets 115 (2014) 135.

[35] D. Abriola et al., Nucl. Data Sheets 107 (2006) 2423.

[36] S. K. Basu et al., Nucl. Data Sheets 111 (2010) 2555.

[37] E. Browne et al., Nucl. Data Sheets 108 (2007) 2173

[38] M. Wang et al., Chinese Phys. C 36 (2012) 1603.

[39] D. Abriola et al., IAEA Consultants Meeting on Beta Delayed Neutron Evaluation, Summary Report, INDC(NDS)-0599, 2011.

[40] H. Bateman, Proc. Camb. Phil. Soc. 16 (1910) 423.

[41] F. James, MINUIT Function Minimization and Error Analysis, CERN Program Library D506, Geneva, 1998.

[42] K. Skrable, Health Physics 27 (1974) 155.

[43] U.C. Bergmann et al., Nucl. Phys. A 714 (2003) 21.

[44] ENDF/B-VII.1, M.B. Chadwick et al., Nucl. Data Sheets 112, 2887 (2011).

45] M.D. Brady, Evaluation and Application of Delayed Neutron Precursor Data, PhD Thesis, Los Alamos National Laboratory, LA-11534-T, 1989.

[46] T. Kawano et al., Phys. Rev. C 78 (2008) 054601.

[47] Compendium of Neutron Spectra and Detector Responses for Radiation Protection Purposes, IAEA, Technical Report Series No. 403, 2001.

[48] A.R. Garcia et al., Validation of the thermal neutron treatment in Geant4, Geant4 Hadronic Group Meeting, 2013. https://indico.cern.ch/event/245281/

[49] J.L. Tain et al., Phys. Rev. Lett. 115 (2015) 062502. 